OPEN ACCESS

Edited by:

Figen Seymen,

Istanbul University, Turkey

Reviewed by:

Bathsheba Turton,

Boston University, United States

Sivakumar Nurvula,

Narayana Dental College and

Hospital, India

*Correspondence:

Bhojraj Nandlal

dr_nandla/@yahoo.com

Specialty section:

This article was submitted to

Pediatric Dentistry,

a section of the journal

Frontiers in Dental Medicine

Received: 08 June 2021

Accepted: 24 August 2021

Published: 11 October 2021

Citation:

Nandlal B, Singh B and Gopi A (2021)

Impact on Utilization and Shift in

Treatment Needs Post-COVID

Lockdown of Pediatric Dentistry in a

Tertiary Care Hospital.

Front. Dent. Med. 2:722292.

doi: 10.3389/fdmed.2021.722292

\section{Impact on Utilization and Shift in Treatment Needs Post-COVID Lockdown of Pediatric Dentistry in a Tertiary Care Hospital}

\author{
Bhojraj Nandlal ${ }^{1 *}$, Birti Singh ${ }^{1}$ and Arun Gopi ${ }^{2}$ \\ 1 Department of Pediatric and Preventive Dentistry, Jagadguru Sri Shivarathreeshwara Dental College and Hospital, \\ Jagadguru Sri Shivarathreeshwara Academy of Higher Education and Research, Mysuru, India, ${ }^{2}$ Department of Community \\ Medicine, Jagadguru Sri Shivarathreeshwara Medical College and Hospital, Jagadguru Sri Shivarathreeshwara Academy of \\ Higher Education and Research, Mysuru, India
}

Background: The COVID-19 pandemic has brought all treatments other than emergencies to a halt. Dental disease, being a multifactorial microbial disease, is capable of progressing to pulpits and its sequelae. The purpose of this study is to predict the impact of utilization of dental services and the progression of treatment needs in children during the lockdown and partial lockdown.

Methods: Outpatient department data from the year 2017-2019 from the Department of Pediatric and Preventive Dentistry, JSS Dental College was collected. A table of treatments provided was prepared. Utilization of services as care-seeking rates at 10, 25 , and $50 \%$ were assumed and modeled corresponding to each stage of the lockdown using linear regression analysis. Dental caries progression was calculated as shifts in treatment needs from permanent restorations to temporary restorations, pulpectomies, or extraction, assuming a 10\% progress to each sequela.

Results: The $p$-values for 10,25 , and $50 \%$ care-seeking rates were $0.021,<0.001$, and $<0.001$, respectively.

Conclusion: The number and severity of cases were predicted to have increased. However, after removal of lockdown, it was noted that the number of patients seeking care was significantly less. The advancement in progression of dental disease further adds to the burden of society and caregivers.

Keywords: disease accumulation, COVID-19, dental caries, epidemiology, pediatric dentistry, disease progression

\section{INTRODUCTION}

Dental caries is a multifactorial microbial disease process caused by the bacterial metabolism of acids diffusing into hard dental tissues and dissolving the mineral content. It is progressive in nature involving the pulp followed by the dissolution of enamel and dentin, which may further progress to pulpitis and its sequelae (1). Caries prevalence in the children of India is as high as $70-80 \%$. Pediatric dentistry is a specialized branch of dentistry catering to the dental needs of children and adolescents as well as that of special patients. 
The novel coronavirus disease-2019 (COVID-19) pandemic due to severe acute respiratory syndrome virus-2 (SARS-CoV2) was declared by $\mathrm{WHO}$ as an international concerning public health emergency on January 30, 2020, with Chinese horshoe bat being the source of origin (2). Its transmission is through droplet spread and contact routes, thereby increasing the risk of cross infection between dental professionals and patients (3). India, having the second largest population in the world with a underdeveloped healthcare system and a majority of the population following an unhygienic lifestyle, managed to follow a strict and early social distancing protocol during the lockdown (4).

The timeline of lockdown in India can be divided into four phases. Phase one began on March 25, 2020 and lasted for 21 days, followed by phase two beginning on April 15, 2020, and ending on May 3, 2020. Phase three and four beginning on May 4, 2020 and May 18, 2020, respectively lasted for 2 weeks each (5). Lockdown restrictions were partially lifted from June 1, 2020 onwards, with services being resumed in a phased manner; this was termed as unlock. Unlock 1.0 lasted the month of June followed by unlock 2.0 announced for the period of July with more ease in restrictions. Unlock 3.0 was further extended for the month of August followed by unlock 4.0.

The Ministry of Health and Family Welfare (MoHFW), Indian Dental Association (IDA), and the Dental Council of India (DCI) issued several guidelines for dental professionals allowing only emergency dental procedures which increased the risk of a patient to death. In children, acute pulpitis and unavoidable dental extractions were treated as "very high" risk. Modifications of dental operatories were also mentioned as per WHO and CDC guidelines. These restrictions ensued during the lockdown and unlock phases in light of the novel COVID-19 pandemic, which caused most activities to halt. This included all elective medical and dental procedures (6). With this regard, many medical and dental institutions including JSS Dental College and Hospital had shut their out-patient department (OPD) as of March 19, 2020 , with only emergency procedures being performed. The beginning of March itself had warranted screening of in-patients and preferable postponement of elective procedures of new OPD patients. The lockdown that began on March 25, 2020 has created a large gap in the demand and supply operations as well as an increasing attention toward optimal patient management and treatment with available resources due to acute shortage of provision of routine dental treatments $(7,8)$. Treatments began upon beginning of the unlock phase in India in June with added infection control measures including personal protective equipment (PPE) kits in dentistry, a field with preexisting high standards of infection control.

The objectives for this study were set to compare the assumed care-seeking rates at 10,25 , and $50 \%$ to the actual rates upon the beginning of unlock period in India commencing on June 1, 2020, as well as to predict the treatment needs due to progression and accumulation of dental caries using linear regression analysis. This was carried out utilizing the average outpatient data for treatments done between the years 2017 and 2019 at the Department of Pediatric and Preventive Dentistry, JSS Dental College and Hospital. Such a prediction model would enable the department to be better prepared for the future in terms of resources and manpower, as literature states that children reporting for treatment are to be considered as potential carriers of the disease unless otherwise proven (9-11). This would further enable health care providers to reduce overcrowding in the outpatient area and improve scheduling of appointments so as to provide better quality patient care services. The care-seeking rates upon the beginning of unlock were to be reviewed as the impact of utilization of services due to the lockdown.

\section{MATERIALS AND METHODS}

This study was done in the Department of Pediatric and Preventive Dentistry at JSS Dental College and Hospital, Mysuru. It was designed as a retrospective observational study. Data used as sample population was the outpatient data records of the years 2017-2020. This data was retrieved from the Medical Records Department of JSS Dental College and Hospital. Analysis has been done cumulatively from 2017 to 2019 as well as individually for each of the treatment procedures.

Data from the department were retrospectively collected from monthly records of the Medical Records Department of the years 2017-2019 and used as database. Average out-patient (OP) numbers of the years 2017 to 2019 were modeled at 10, 25, and $50 \%$ using linear regression analysis to predict the expected number of treatments for the year 2020. These were compared with the actual OP numbers of 2020 coming to a halt during the lockdown and inflow during the unlock phase post the lockdown. These numbers represent the care-seeking rates of the pediatric population.

Data of the various treatment procedures done in the Department of Pediatric and Preventive Dentistry were tabulated for the years 2017-2019 on Microsoft excel spreadsheet into nine categories including temporary restorations (TEMP), indirect pulp capping (IPC), direct pulp capping (DPC), glass ionomer cement restorations (GIC), light cure composite restorations (LC), pulpectomies (PULPEC), permanent teeth root canal treatments (RCT), extractions of deciduous teeth (EXT), and extractions of permanent teeth (EXT) from the database. Data on cleft lip and palate management, orthodontic treatments, and trauma have been excluded from this analysis. The average number of treatments done from 2017 to 1019 were used in calculating the predictions of this study (Table 1). A linear regression analysis has been done to predict the expected number of treatment procedures during the lockdown and postlockdown period.

This research was approved by the Institutional Ethics Committee of JSS Dental College and Hospital (JSSDCH IEC Research Protocol No: 27/2020).

\section{CALCULATIONS}

Calculations for acquiring expected OP numbers were made assuming a possible turn up of 10,25 , and $50 \%$ corresponding to the end of May (lockdown phases one and two), June 
TABLE 1 | Average OP statistics of the Department of Pediatric and Preventive Dentistry, JSS Dental College and Hospital, Mysuru.

\begin{tabular}{|c|c|c|c|c|c|c|c|c|c|c|c|c|}
\hline Treatment & January & February & March & April & May & June & July & August & September & October & November & December \\
\hline New OP & 201 & 138 & 152 & 320 & 341 & 178 & 200 & 153 & 147 & 226 & 162 & 184 \\
\hline Old OP & 787 & 722 & 486 & 725 & 850 & 619 & 592 & 501 & 395 & 507 & 438 & 582 \\
\hline Total OP & 985 & 860 & 638 & 1,046 & 1,191 & 797 & 791 & 661 & 543 & 731 & 601 & 767 \\
\hline Treated OP & 809 & 687 & 690 & 840 & 1,068 & 806 & 774 & 638 & 471 & 608 & 592 & 628 \\
\hline $\begin{array}{l}\text { Temporary Restorations } \\
\text { (TEMP) }\end{array}$ & 60 & 59 & 58 & 72 & 62 & 60 & 49 & 55 & 35 & 74 & 86 & 113 \\
\hline $\begin{array}{l}\text { Indirect pulp capping } \\
\text { (IPC) }\end{array}$ & 38 & 29 & 26 & 30 & 52 & 32 & 22 & 27 & 19 & 23 & 23 & 36 \\
\hline $\begin{array}{l}\text { Direct pulp capping } \\
\text { (DPC) }\end{array}$ & 3 & 3 & 1 & 7 & 4 & 5 & 3 & 2 & 3 & 1 & 1 & 4 \\
\hline $\begin{array}{l}\text { Glass ionomer cement } \\
(\mathrm{GIC})\end{array}$ & 170 & 151 & 137 & 210 & 247 & 168 & 188 & 161 & 109 & 143 & 125 & 159 \\
\hline Light cure composite (LC) & 44 & 33 & 41 & 28 & 42 & 33 & 32 & 20 & 18 & 13 & 17 & 26 \\
\hline Pulpectomies (PULPEC) & 23 & 42 & 43 & 40 & 53 & 48 & 33 & 39 & 25 & 30 & 41 & 63 \\
\hline $\begin{array}{l}\text { Root canal treatment } \\
(\mathrm{RCT}) \text {-Permanent teeth }\end{array}$ & 36 & 33 & 30 & 30 & 29 & 29 & 37 & 29 & 20 & 29 & 26 & 36 \\
\hline $\begin{array}{l}\text { Extractions-deciduous } \\
\text { teeth }(E X T)\end{array}$ & 105 & 75 & 80 & 122 & 138 & 93 & 90 & 73 & 55 & 76 & 67 & $82^{a}$ \\
\hline
\end{tabular}

${ }^{a}$ Average OP statistics, Department of Pediatric and Preventive Dentistry, JSSDCH, Mysuru.

TABLE 2 | Initial baseline prediction and possible care-seeking rates.

\begin{tabular}{|c|c|c|c|c|c|c|c|c|c|}
\hline \multirow{2}{*}{$\begin{array}{l}\text { Treatment } \\
\text { Turn up }\end{array}$} & \multicolumn{3}{|c|}{ May (Lockdown phases 1 and 2) } & \multicolumn{3}{|c|}{ June (Lockdown phases 3 and 4) } & \multicolumn{3}{|c|}{ September (Unlock 1.0-3.0) } \\
\hline & $10 \%$ & $25 \%$ & $50 \%$ & $10 \%$ & $25 \%$ & $50 \%$ & $10 \%$ & $25 \%$ & $50 \%$ \\
\hline New OP & 81 & 203 & 407 & 99 & 248 & 496 & 149 & 373 & 745 \\
\hline Old OP & 206 & 515 & 1,031 & 268 & 670 & 1,340 & 417 & 1,042 & 2,084 \\
\hline Total OP & 287 & 719 & 1,437 & 367 & 918 & 1,836 & 567 & 1,417 & 2,833 \\
\hline Temporary Restorations (TEMP) & 19 & 48 & 96 & 25 & 63 & 126 & 39 & 98 & 195 \\
\hline Indirect pulp capping (IPC) & 11 & 27 & 54 & 14 & 35 & 70 & 21 & 52 & 104 \\
\hline Direct pulp capping (DPC) & 1 & 3 & 6 & 2 & 4 & 8 & 2 & 6 & 12 \\
\hline Glass ionomer cement (GIC) & 60 & 149 & 298 & 76 & 191 & 382 & 122 & 305 & 610 \\
\hline Light cure composite (LC) & 11 & 28 & 56 & 14 & 36 & 72 & 21 & 54 & 107 \\
\hline Pulpectomies (PULPEC) & 14 & 34 & 68 & 18 & 46 & 92 & 28 & 70 & 141 \\
\hline Root canal treatment (RCT) - Permanent teeth & 9 & 22 & 45 & 12 & 30 & 59 & 21 & 51 & 102 \\
\hline Extractions - deciduous teeth (EXT) & 34 & 85 & 170 & 43 & 108 & 217 & 65 & 163 & 326 \\
\hline
\end{tabular}

The initial baseline prediction and possible care-seeking rates as observed as utilization of pediatric dental services by patients modeled at 10,25, and $30 \%$ care-seeking rates.

(lockdown phases three and four), and September (unlock 1.03.0), respectively (Table 2).

Dental caries progression was calculated as shifts in treatment needs, modeled on the following criteria:

1) A $10 \%$ progression of the treatments took place every month. A $10 \%$ progression was assumed as per observations of previous data from the department revealing an $8-10 \%$ shift in treatment needs.

2) The disease progressed such that there was a $10 \%$ shift in progression of the disease to the next treatment need as per the flow chart in Figure 2 with the following: 10\% temporary restoration progressed to IPC; $10 \%$ IPC progressed to DPC; $10 \%$ DPC to PULPEC and RCT. Ten percent GIC and LC progressed to temporary restorations; $10 \%$ of which moved to IPC followed by DPC and further followed by PULPEC and RCT. Ten percent of PULPEC and RCT shifted to deciduous and permanent teeth extractions, respectively (Figure 1).

3) The elective procedures were restricted in March 2020 while undertaking only emergency treatment as per government interim advisories; hence calculations on progression were made from March onwards.

4) Treatments were resumed upon unlock beginning in June 2020 with additional personal protective equipment.

The progression of treatment needs was calculated using linear regression analysis for the months of May, June, and September which corresponded with the end of lockdown phases one and 


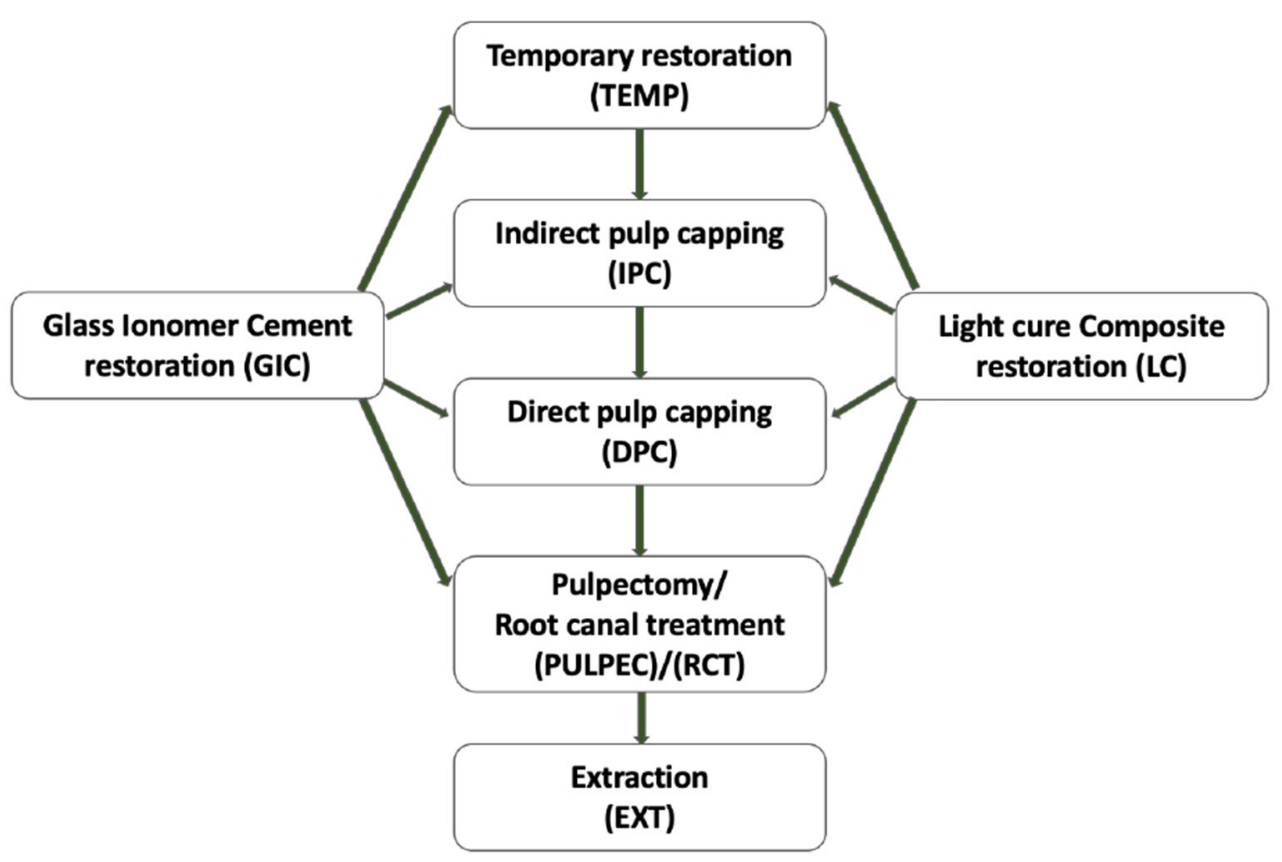

FIGURE 1 | Progression of Treatment Needs. The progression of dental caries as shift in treatment needs: 10\% temporary restoration progressed to IPC; $10 \%$ IPC progressed to DPC; 10\% DPC to PULPEC and RCT. 10\% GIC and LC progressed to temporary restorations; $10 \%$ of which moved to IPC followed by DPC and further followed by PULPEC and RCT. Ten percent of PULPEC and RCT shifted to deciduous and permanent teeth extractions respectively.

TABLE 3 | Prediction of progression of treatment needs in pediatric dentistry.

\begin{tabular}{|c|c|c|c|c|c|c|}
\hline Treatment & $\begin{array}{c}\text { May average } \\
\text { cases }\end{array}$ & $\begin{array}{c}\text { Expected } \\
\text { May } 2020 \\
\text { cases }\end{array}$ & $\begin{array}{c}\text { June } \\
\text { average } \\
\text { cases }\end{array}$ & $\begin{array}{c}\text { Expected } \\
\text { June } 2020 \\
\text { cases }\end{array}$ & $\begin{array}{c}\text { September } \\
\text { average } \\
\text { cases }\end{array}$ & $\begin{array}{l}\text { Expected } \\
\text { September } \\
2020 \text { cases }\end{array}$ \\
\hline Temporary Restorations (TEMP) & 62 & 249 & 60 & 303 & 35 & 597 \\
\hline Indirect pulp capping (IPC) & 52 & 189 & 32 & 212 & 19 & 506 \\
\hline Direct pulp capping (DPC) & 4 & 74 & 5 & 83 & 3 & 277 \\
\hline Glass ionomer cement (GIC) & 247 & 390 & 168 & 559 & 109 & 425 \\
\hline Light cure composite (LC) & 42 & 70 & 33 & 106 & 18 & 70 \\
\hline Pulpectomies (PULPEC) & 53 & 157 & 48 & 223 & 25 & 372 \\
\hline Root canal treatment (RCT) - Permanent teeth & 29 & 78 & 29 & 110 & 20 & 152 \\
\hline Extractions - deciduous teeth (EXT) & 138 & 357 & 93 & 447 & 55 & 742 \\
\hline Extractions - permanent teeth (EXT) & 0 & 12 & 0 & 9 & 0 & 52 \\
\hline
\end{tabular}

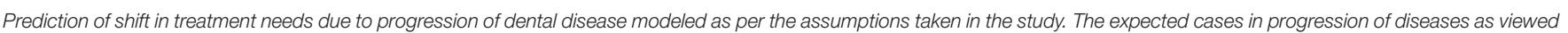
due to accumulation and underutilization of services corresponding to various stages of the lockdown and unlock in India.

two; lockdown phases three and four; and unlock 1.0-3.0 in India, respectively. Further calculations were completed using Microsoft excel spreadsheet (Table 3). The calculations were then compared with the actual care-seeking rates upon beginning of unlock.

\section{RESULTS}

The average and expected care-seeking rates obtained on performing linear regression analysis of the Department of Pediatric and Preventive Dentistry are presented in Table 2, and comparison with the actual care-seeking rates upon the beginning of unlock period are presented in Figure 2, respectively. It can be noted that the expected care-seeking rates at 10,25 , and $50 \%$ for each month amount to a very high value that further increases as the number of untreated cases of previous months get accumulated. However, the actual careseeking behavior can be observed to be lesser than the lowest assumed value of $10 \%$, with slight improvement as the months progressed. Thus, it can be concluded that the number of patients seeking care and utilizing dental services are significantly lesser than those predicted to report to the pediatric department. 


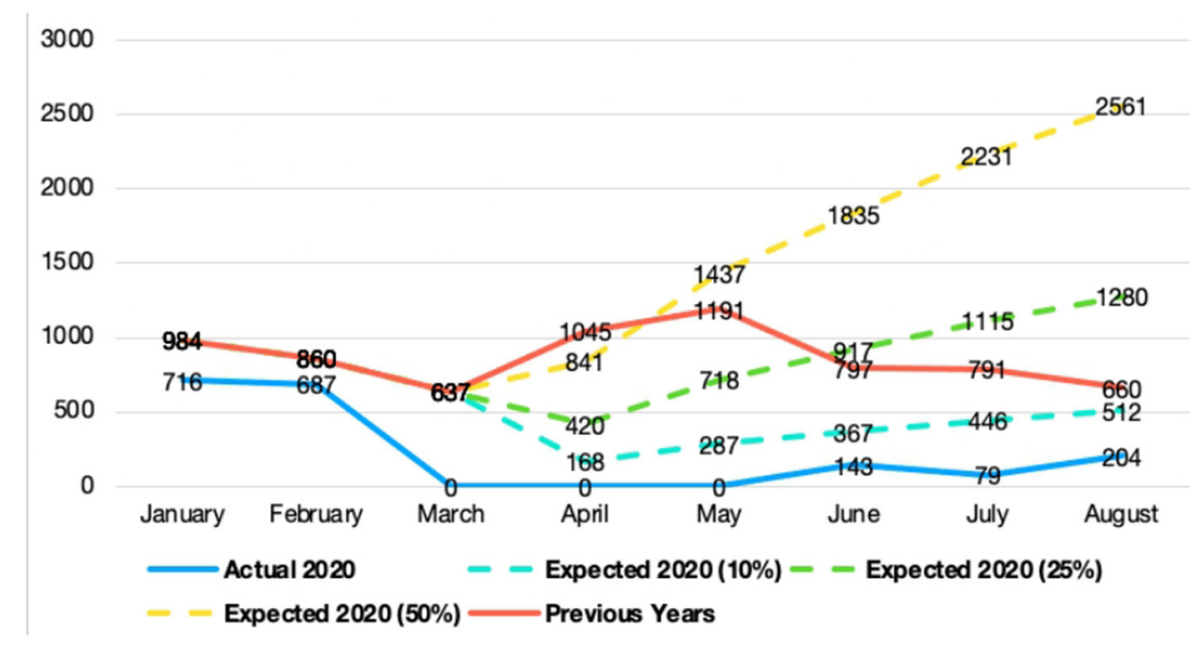

FIGURE 2 | Predicted and actual care seeking rates post the COVID Lockdown. The predicted and actual care-seeking rates as seen as outpatient numbers in the Department of Pediatric and Preventive Dentistry, JSS Dental College and Hospital, Mysuru. Previous year cases are the average values of OP numbers from the years 2017 to 2019. The predicted cases are modeled at 10, 25, and 50\% using linear regression analysis. These are compared with the OP numbers of 2020 coming to a halt during the lockdown and inflow during the unlock phase post the lockdown. A statistically significant variation was observed in the number of actual and expected number of out-patients with the p-values $0.021,<0.001$, and $<0.001$ for 10,25 , and $50 \%$, respectively.

Chi square test for trend was performed to compare the actual and expected care-seeking rates (OP numbers) of 10, 25, and $50 \%$. A statistically significant variation was observed in the number of actual and expected number of out-patients seeking dental treatment.

A tabulation of a shift in progression of treatment needs to the next sequela of pulpitis modeled on the predictive progression and accumulation of treatment procedure has been presented in Table 3 for each procedure. A graph representing the progression and accumulation of treatment procedures for each of the lockdown and unlock stages considered in this study has been prepared (Figure 3). The prediction of progression of dental caries as the shift in treatment needs are noted to have increased with every stage of the lockdown, possibly due to the unavailability of treatment during the lockdown (expected 2020 progression) and underutilization of services post the lockdown (expected 2020 due to other treatment needs). Hence, this shift can be attributed to the unavailability (due to restriction of elective procedures by national and international advisories) and underutilization of dental services during the lockdown period.

An increase in the number of temporary restorations, indirect pulp capping, direct pulp capping, pulpectomies, and root canal treatments is seen due to the predictive progression and also the accumulation of newer cases at the end of each month. Whereas, an increase in the treatments requiring glass ionomer cement and light cure composite restorations occurred only due to the accumulation at the end of each month and not due to progression from any other treatment need. Extraction of deciduous and permanent teeth are also noted to have increased in number owing to their progression from pulpectomies and root canal treatments, respectively.

\section{DISCUSSION}

This study aims to predict the care-seeking rates, utilization, progression, and accumulation of treatment need in children in a tertiary care hospital during the lockdown and partial lockdown period in a tertiary dental hospital in Mysore city, India.

As per the data processed in this study, there has been a notable underutilization of all dental services in a tertiary care hospital setting post the lockdown due to the coronavirus pandemic. In the Department of Pediatric and Preventive Dentistry, an increase in the utilization of dental manpower was expected post the lockdown. However, the actual care-seeking rates are viewed to have dropped drastically owing primarily to the inherent fear and stigma associated with the virus spread. It was noted in China that despite the large scale transmission of the disease, the urgent dental treatment had decreased by only $38 \%$, indicating the need for urgent dental treatment as an essential service (12).

In the pediatric setting, children are assumed to be potential carriers of the disease until proven otherwise thereby causing further underutilization of services (11). This is despite the lack of evidence that aerosols generated during dental treatment can lead to transmission of the virus (13). The fear of the spread of COVID due to aerosols generated during dental procedures caused several national and international bodies including WHO, CDC, IDA, DCI, and MoHFW to restrict all elective dental procedures. The risk of caries in children has increased compared with previous decades because of high sugar diets, sedentary lifestyles, and chronic health conditions (14). This may impact the pediatric population with a greater number of urgent dental treatments required for advanced dental conditions, including acute irreversible pulpitis and abscesses. 


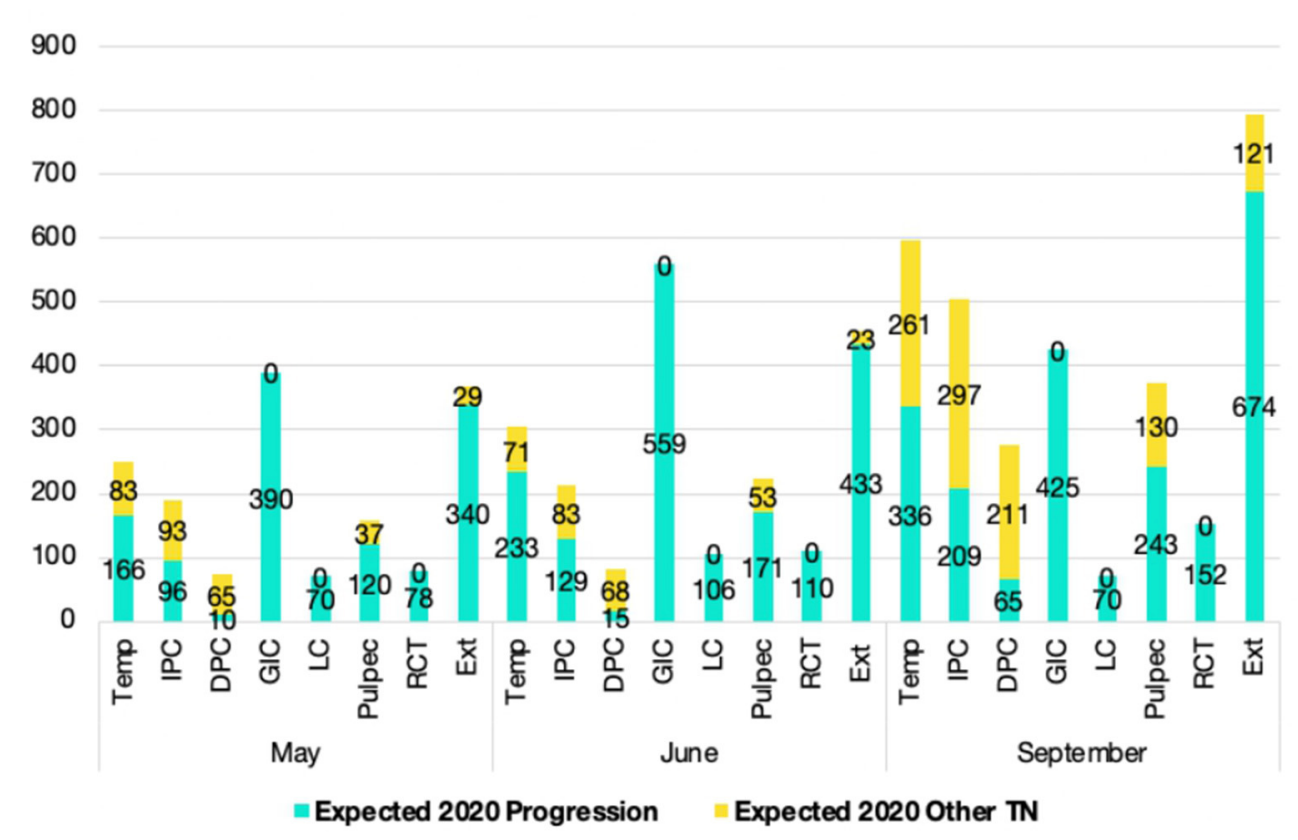

FIGURE 3 | Prediction of shift in progression of treatment needs. The prediction of progress of dental caries as shift in treatment needs are noted to have increased with every stage of the lockdown.

The shift in progression of treatment needs may have increased due to progression, accumulation as well as newer incoming cases owing to the inherent progressive nature of dental caries. However, various problems are associated with such an increase in number of cases advancing to pulpectomies as they require a specialist for the provision of treatment. This would create a load for the limited specialists, including post graduate students and staff. Further referral from local centers or private practitioners hesitant to work during an ongoing pandemic might increase the load.

According to an online survey conducted in the country, the preparedness of Indian dental professionals seemed inadequate during and after the pandemic due to professional as well as medico-legal concerns (15). A survey assessing the knowledge, attitudes, practices of practitioners regarding the current pandemic across various countries, including most Asian ones, found dentists having good knowledge and practice scores. These scores were found to be significantly associated with years of practice and qualifications (16). Another study done in the Indian scenario among dental health professionals to assess the knowledge, awareness, and hygiene practices showed notable deficiencies regarding some vital aspects of COVID-19. The authors recommended an urgent need for health education and training programs for improving the knowledge of dentists (17). A similar cross-sectional study was carried out to evaluate the knowledge, attitude, and practices along with the perceived barriers in practicing dentistry during the pandemic elicited poor median score for "knowledge." However, the "attitude" median score was observed to be good and "practices scores" for participants over 40 years of age were significantly better (18). These surveys indicate that health care professionals treating and not treating COVID-19 positive patients seem unprepared and urge the policymakers for better protocols. A model to assess the impact of lockdown and the disease transmission in India suggested a reduction in the transmission of the COVID-19 virus as well as in the burden on the health care system (19). At the time of writing this paper, we did not come across any study with respect to the accumulation or progression of disease states in pediatric dentistry.

A literature review on the current clinical practices in pediatric dentistry post COVID 19 concluded that behavior management would include a thorough explanation with regard to the professional dressing up in PPEs (20). Treatments avoiding aerosol procedures and those that incorporated minimally invasive procedures were sought to be preferred wherever possible (20). Hence, a change in the way routine pediatric dental treatments are managed may be seen with more focus on minimal interventional dentistry, atraumatic restorative treatments, application of fissure sealants, usage of silver diamine fluoride, and Hall technique $(21,22)$.

The increased number of cases in the dental operatory warrants a proper disinfection protocol with special personal protective gear for treatments generating aerosols and those not generating aerosols (21). Therefore, a structured pretreatment, treatment and posttreatment protocol need to be followed with proper hand hygiene, oral hygiene, and tele-dentistry practices for future implications $(3,23)$. An increased emphasis on disinfection and prevention of cross contamination with the use 
of preprocedural mouth rinses and environmental disinfection is warranted $(20,24)$. The Hospital was fully geared as per the interim guidance by WHO and other guidelines by MoHFW with PPEs, UV and chemical sterilizations, screening procedures, and triage. The dental hospital was also backed up with the Medical Hospital in case of any emergency. Despite the increased preparedness, a fall in the care-seeking rates was noted in this study.

\section{CONCLUSION}

Although there is plenty of literature around the biosafety of dental practices in pediatric dentistry post COVID, none provides a perspective on the increased suffering due to the progression of dental caries and the pain it can cause. This in turn can have a decreased quality of life in children due to perceived pain leading to chewing difficulties. From this study, it can be safely concluded that the care-seeking rates after removal of lockdown were lesser than the assumed $10 \%$ values in a tertiary health care hospital in Mysuru, a city in Southern Karnataka. The progression of dental disease as modeled in this study is significantly high with underutilization of services. This adds to the burden on society and the caregivers due to the progression of disease states and the increased cost of treatments. Thus, we urge the medical practitioners to help make patients aware of safe utilization of dental services at the appropriate time before further advancement of the disease occurs, leading to disability in children. The fear of cross infection is also advocated to be limited due to the high standards of infection control followed in the field of dentistry, which have improved further to avoid cross infection of the coronavirus as well.

\section{REFERENCES}

1. Featherstone JDB. Dental caries: a dynamic disease process. Aust Dent J. (2008) 53:286-91. doi: 10.1111/j.1834-7819.2008. 00064.x

2. Chen ZM, Fu JF, Shu Q, Chen YH, Hua CZ, Li FB, et al. Diagnosis and treatment recommendations for pediatric respiratory infection caused by the 2019 novel coronavirus. World J Pediatr. (2020) 16:240-6. doi: 10.1007/s12519-020-00345-5

3. Bhanushali P, Katge F, Deshpande S, Chimata VK, Shetty S, Pradhan D. COVID-19: changing trends and its impact on future of dentistry. Int J Dent. (2020) 2020:8817424. doi: 10.1155/2020/8817424

4. Paital B, Das K, Parida SK. Inter nation social lockdown versus medical care against COVID-19, a mild environmental insight with special reference to India. Sci Total Environ. (2020) 728:138914. doi: 10.1016/j.scitotenv.2020.138914

5. Rammohan A, Rela M. COVID-19: could India still escape?. J Glob Health. (2020) 10:1-4. doi: 10.7189/jogh.10.010372

6. The Lancet. India Under COVID-19 Lockdown. Lancet. (2020) 395:1315. doi: 10.1016/S0140-6736(20)30938-7

7. WHO. Coronavirus Disease Case Report - 18 May 2020. World Health Organization (2020) 2633 p.

8. Sharma N, Hasan Z, Velayudhan A, A EM, Mangal DK, Gupta SD. Personal protective equipment: challenges and strategies to combat COVID19 in India: a narrative review. J Health Manag. (2020) 22:157-68. doi: $10.1177 / 0972063420935540$

\section{LIMITATIONS}

i. Gender and age distribution of data was missing electronically.

ii. Shift in treatment needs was assumed to be $10 \%$, which may not be accurate due to the absence of direct literature on the same.

iii. Further studies are required to generalize the results to a global population.

\section{DATA AVAILABILITY STATEMENT}

The raw data supporting the conclusions of this article will be made available by the authors, without undue reservation.

\section{ETHICS STATEMENT}

The studies involving human participants were reviewed and approved by the Institutional Ethics Committee of JSS Dental College and Hospital (JSSDCH IEC Research Protocol No: 27/2020). Written informed consent was not required for this study, in accordance with the local legislation and institutional requirements.

\section{AUTHOR CONTRIBUTIONS}

All authors listed have made a substantial, direct and intellectual contribution to the work, and approved it for publication.

\section{FUNDING}

This work was supported by International Foundation for Education and Self Help (DSC-08031581025PRD).

9. Mallineni S, Innes PN, Raggio DP, Robertson MD, Jayaraman J. Coronavirus disease (COVID-19): characteristics in children and considerations for dentists providing their care. Int J Paediatr Dent. (2020) 30:245-50. doi: 10.1111/ipd.12653

10. Mathur R. Ethics preparedness for infectious disease outbreaks research in India: a case for novel coronavirus disease 2019. Indian J Med Res. (2020) 151:124-31. doi: 10.4103/ijmr.IJMR_463_20

11. Al-Halabi M, Salami A, Alnuaimi E, Kowash M, Hussein I. Assessment of paediatric dental guidelines and caries management alternatives in the post COVID-19 period. A critical review and clinical recommendations. Eur Arch Paediatr Dent. (2020) 21:543-56. doi: 10.1007/s40368-020-00547-5

12. Guo H, Zhou Y, Liu X, Tan J. The impact of the COVID-19 epidemic on the utilization of emergency dental services. J Dent Sci. (2020) 15:564-7. doi: $10.1016 /$ j.jds.2020.02.002

13. Epstein JB, Chow K, Mathias R. Dental procedure aerosols and COVID-19. Lancet Infect Dis. (2021) 21:e73. doi: 10.1016/S1473-3099(20)30636-8

14. Lamont RJ, Egland PG. Dental caries. Mol Med Microbiol. (2014) 3:945-55. doi: 10.1016/B978-0-12-397169-2.00052-4

15. Kinariwala N, Perera Samaranayake L, Perera I, Patel Z. Concerns and fears of indian dentists on professional practice during the coronavirus disease2019 (COVID-19) pandemic. Oral Dis. (2020). 730-732. doi: 10.1111/odi. 13459

16. Kamate SK, Sharma S, Thakar S, Srivastava D, Sengupta K, Hadi AJ, et al. Assessing knowledge, attitudes and practices of dental practitioners regarding the covid-19 pandemic: a multinational study. Dent Med Probl. (2020) 57:117. doi: $10.17219 / \mathrm{dmp} / 119743$ 
17. Gambhir RS, Dhaliwal JS, Aggarwal A, Anand S. Covid-19: a survey on knowledge, awareness and hygiene practices among dental health professionals in an Indian scenario. Rocz Państwowego Zakładu Hig. (2020) 71:223-9. doi: 10.32394/rpzh.2020.0115

18. Singh KT, Mishra G, Shukla AK, Behera S, Tiwari AK, Panigrahi S, et al. Preparedness among dental professionals towards COVID-19 in India. Pan Afr Med J. (2020) 36:1-7. doi: 10.11604/pamj.2020.36.108.23694

19. Ambikapathy B, Krishnamurthy K. Mathematical modelling to assess the impact of lockdown on COVID-19 transmission in India: model development and validation. J Med Internet Res. (2020) 6:e19368. doi: 10.2196/preprints. 19368

20. Sales SC, Meyfarth S, Scarparo A. The clinical practice of Pediatric Dentistry post-COVID-19: the current evidences. Pediatr Dent J. (2021) 31:25-32. doi: 10.1016/j.pdj.2021.01.002

21. Cianetti S, Pagano S, Nardone M, Lombardo G. Model for taking care of patients with early childhood caries during the SARS-CoV-2 pandemic. Int J Environ Res Public Health. (2020) 17:1-17. doi: 10.3390/ijerph17113751

22. BaniHani A, Gardener C, Raggio DP, Santamaría RM, Albadri S. Could COVID-19 change the way we manage caries in primary teeth? Current implications on Paediatric Dentistry. Int J Paediatr Dent. (2020) 30:523-5. doi: 10.1111/ipd.12690

23. World Health Organization. Considerations for the Provision of Essential Oral Health Services in the Context of COVID-19: Interim Guidance. (2020). 1-5 p.
24. de Amorim LM, Maske TT, Ferreira SH, Dos Santos RB, Feldens CA, Kramer PF. New post-COVID-19 biosafety protocols in pediatric dentistry. Pesqui Bras Odontopediatr Clin Integr. (2020) 20:1-9. doi: 10.1590/pboci. 2020.117

Conflict of Interest: The authors declare that the research was conducted in the absence of any commercial or financial relationships that could be construed as a potential conflict of interest.

Publisher's Note: All claims expressed in this article are solely those of the authors and do not necessarily represent those of their affiliated organizations, or those of the publisher, the editors and the reviewers. Any product that may be evaluated in this article, or claim that may be made by its manufacturer, is not guaranteed or endorsed by the publisher.

Copyright (C 2021 Nandlal, Singh and Gopi. This is an open-access article distributed under the terms of the Creative Commons Attribution License (CC BY). The use, distribution or reproduction in other forums is permitted, provided the original author(s) and the copyright owner(s) are credited and that the original publication in this journal is cited, in accordance with accepted academic practice. No use, distribution or reproduction is permitted which does not comply with these terms. 